\title{
Statefinder Diagnostic for Binary Mixture of Anisotropic Dark Energy and Perfect Fluid in Bianchi Type-IX Universe
}

\author{
H. R. Ghate ${ }^{1}$, Atish S. Sontakke ${ }^{2}$ \\ ${ }^{1,2}$ Department of Mathematics, Jijamata Mahavidyalaya, Buldana (India)-443001
}

\begin{abstract}
The new Bianchi type-IX cosmological models with binary mixture of perfect fluid and anisotropic dark energy have been studied. The unique solution of field equations is obtained by assuming that the energy conservation equation of the perfect fluid and dark energy vanishes separately together with a special law for the mean Hubble parameter (Berman, 1983) which yields a constant value of the deceleration parameter and generates two types of solutions, one is of power law type and other is of the exponential type. To have a general description of an anisotropic dark energy component in terms of its equation of state $\omega^{(d e)}$, a skewness parameters $\gamma$ have been introduced. The statefinder diagnostic pair (i.e. $\{r, s\}$ parameter) is used to characterize different phases of the universe. The various geometric and kinematic properties of the model and the behavior of the anisotropy of the dark energy have been discussed.
\end{abstract}

Keywords - Anisotropic Dark Energy, Perfect Fluid, Bianchi type-IX Universe, Statefinder parameters.

\section{Introduction}

In 1998, two teams have independently studied distant type Ia Supernovae (SNe Ia) and observed that, the universe is experiencing an accelerated expansion [1-2]. These remarkable findings were confirmed later from the studies of supernovae and independent evidences from cluster of galaxies, large scale structure [3-4] and the cosmic microwave background (CMB) [5-6]. In Einstein's general relativity, for such type of acceleration, one needs to introduce a component to the matter distribution of the universe with a large negative pressure. This component is usually referred as 'Dark Energy' (DE). The DE occupies about $73 \%$ of the total energy of the universe, while dark matter occupies $23 \%$ and the usual baryonic matter is $4 \%$. DE is described phenomenologicaly by an equation of state (EoS) $p=\omega \rho$ where $p$ and $\rho$ are the pressure and energy

density of DE and $\omega$ is an EoS parameter which is useful to describe cosmic acceleration. A number of authors [7-15] have studied binary mixture of perfect fluid (PF) and anisotropic DE for Bianchi type-I, II, III, $\mathrm{V}$, VI0, plane symmetric, kaluza-klein as well as higher dimensional cosmological models too. Recently, the anisotropic dark energy has been studied for Bianchi type-IX space-time [16].

The theoretical arguments and recent experimental data supports the existence of an anisotropic phase that approaches anisotropy. This is because we consider models of the universe with an anisotropic background. Bianchi type homogeneous models are the simplest of the anisotropic models. These models also used to examine the role of certain anisotropic sources during the formation of the large scale structure as we see in the universe today. Bianchi type-IX cosmological models are very popular for relativistic studies. They have solutions of type Robertson-Walker universe with positive curvature, the de-Sitter universe and Taub-NUT solutions. These models are not only anisotropic but also allow expansion, rotation and shear. Many authors [1625] have studied different aspects of Bianchi type-IX cosmological models in various theories of gravitation. Recently, Bianchi type-IX DE model in Brans-Dicke theory of gravitation has been studied [26].

In the last few years, high red-shift supernova experiments had dramatically increased the quality of cosmological observational data. There are many DE models proposed recently to explain the cosmic acceleration. From the observations, we see that there is a variation in the properties of DE depending on the cosmological model. In order to differentiate among different forms of DE, Sahni et al. [27] proposed a new geometrical diagnostic pair of statefinders parameters $\{r, s\}$ which are defined as

$$
r=\frac{\dddot{R}}{R H^{3}} \text { and } s=\frac{r-1}{3(q-1 / 2)},
$$

Where $H$ is the Hubble parameter, $R$ is an average scale factor and $q$ is the deceleration parameter. The parameter $r$ forms the next step in the hierarchy of geometrical cosmological parameters after the Hubble parameter $H$ and the deceleration parameter $q$, while $s$ is a linear combination of $q$ and $r$ chosen in such a way that it does not depend upon the DE density. The statefinder pair is calculated for a number of existing 
models of DE [28-38] having both constant and variable $\omega$. Hence, we are motivated to study Bianchi type-IX DE model using this method of Statefinder diagnostic.

In the present paper, we have investigated new Bianchi type-IX cosmological models with binary mixture of anisotropic DE and perfect fluid. The statefinder diagnostic pair $\{r, s\}$ is adopted to characterize different phases of the universe. The geometrical and physical behaviors of the model are also discussed.

\section{Metric And The Field Equations:}

Bianchi type-IX metric is considered in the form

$$
d s^{2}=-d t^{2}+a^{2} d x^{2}+b^{2} d y^{2}+\left(b^{2} \sin ^{2} y+a^{2} \cos ^{2} y\right) d z^{2}-2 a^{2} \cos y d x d z,
$$

where $a, b$ are scale factors and are functions of cosmic time $t$.

The model has one transverse direction $x$, and two equivalent longitudinal directions $y$ and $z$. In natural units $(8 \pi G=1, c=1)$, Einstein's field equations in case of a binary mixture of PF and anisotropic DE components are

$$
G_{i j}=R_{i j}-\frac{1}{2} g_{i j} R=-\left({ }^{p f} T_{i j}+{ }^{d e} T_{i j}\right)
$$

with

$$
\begin{aligned}
& { }^{p f} T_{i}^{j}=\operatorname{diag}\left[-\rho^{(p f)}, p^{(p f)}, p^{(p f)}, p^{(p f)}\right] \\
& \quad=\operatorname{diag}\left[-1, \omega^{(p f)}, \omega^{(p f)}, \omega^{(p f)}\right] \rho^{(p f)},
\end{aligned}
$$

and

$$
\begin{aligned}
{ }^{d e} \boldsymbol{T}_{i}^{j} & =\operatorname{diag}\left[-\rho^{(d e)}, p_{x}^{(d e)}, p_{y}^{(d e)}, p_{z}^{(d e)}\right] \\
& =\operatorname{diag}\left[-1, \omega_{x}^{(d e)}, \omega_{y}^{(d e)}, \omega_{z}^{(d e)} \mid \rho^{(d e)},\right. \\
& =\operatorname{diag}\left[-1, \omega^{(d e)},\left(\omega^{(d e)}+\gamma\right),\left(\omega^{(d e)}+\gamma\right)\right] \rho^{(d e)},
\end{aligned}
$$

where, $g_{i j}$ is the metric potentials with $g_{i j} u^{i} u^{j}=1 ; u^{i}$ is the flow vector; $R_{i j}$ is the Ricci tensor; $R$ is the Ricci scalar; $\rho^{(p f)}$ and $\rho^{(d e)}$ are the energy densities of PF and DE components respectively; $\omega^{(p f)}$ is the EoS parameter of PF with $\omega^{(p f)} \geq 0$ and $\omega^{(d e)}$ is the EoS parameter of DE; $\omega_{x}^{(d e)}, \omega_{y}^{(d e)}$ and $\omega_{z}^{(d e)}$ are the deviation-free EoS parameter of the DE on the $x, y$ and $z$ axes, respectively. $\gamma$ is the deviation-free EoS parameters of the DE. Here $\omega$ and $\gamma$ are not necessarily constants and can be function of the cosmic time $t$.

The Einstein's field equations (3) for metric (2) with the help of equations (3) and (5) can be written as

$$
\begin{aligned}
& 2 \frac{\dot{a}}{a} \frac{\dot{b}}{b}+\frac{\dot{b}^{2}}{b^{2}}+\frac{1}{b^{2}}-\frac{a^{2}}{4 b^{4}}=\rho^{(p f)}+\rho^{(d e)} \\
& 2 \frac{\ddot{b}}{b}+\frac{\dot{b}^{2}}{b^{2}}+\frac{1}{b^{2}}-\frac{3 a^{2}}{4 b^{4}}=-\omega^{(p f)} \rho^{(p f)}-\omega^{(d e)} \rho^{(d e)} \\
& \frac{\ddot{a}}{a}+\frac{\ddot{b}}{b}+\frac{\dot{a}}{a} \frac{\dot{b}}{b}+\frac{a^{2}}{4 b^{4}}=-\omega^{(p f)} \rho^{(p f)}-\left(\omega^{(d e)}+\gamma\right) \rho^{(d e)},
\end{aligned}
$$

where over dot $\left({ }^{\circ}\right)$ denotes the differentiation with respect to $t$.

The Bianchi identity is given by

This yields:

$$
G_{; j}^{i j}={ }^{p f} T_{; j}^{i j}+{ }^{d e} T_{; j}^{i j}=0
$$

$$
\dot{\rho}^{(p f)}+\left(1+\omega^{(p f)}\right) \rho^{(p f)}\left(\frac{\dot{a}}{a}+2 \frac{\dot{b}}{b}\right)+\dot{\rho}^{(d e)}+\left(1+\omega^{(d e)}\right) \rho^{(d e)}\left(\frac{\dot{a}}{a}+2 \frac{\dot{b}}{b}\right)+2 \gamma \rho^{(d e)} \frac{\dot{b}}{b}=0 .
$$

The directional Hubble parameters in the directions of $\mathrm{x}, \mathrm{y}$ and $\mathrm{z}$ axes for the metric (2) are defined as

$$
H_{x}=\frac{\dot{a}}{a}, H_{y}=H_{z}=\frac{\dot{b}}{b} \text {. }
$$


The mean Hubble parameter is

$$
H=\frac{1}{3} \frac{\dot{V}}{V}=\frac{1}{3}\left(\frac{\dot{a}}{a}+2 \frac{\dot{b}}{b}\right),
$$

where the spatial volume of the universe is

$$
V=a b^{2} \text {. }
$$

The anisotropy of the expansion can be parameterized after defining the directional Hubble parameters and the mean Hubble parameter of the expansion.

The anisotropic parameter of the expansion is defined as

$$
\Delta=\frac{1}{3} \sum_{i=1}^{3}\left(\frac{H_{i}-H}{H}\right)^{2}
$$

(14) where $H_{i}(i=1,2,3)$ represent the directional Hubble parameters in the directions of $\mathrm{x}, \mathrm{y}$ and $\mathrm{z}$ respectively. $\Delta=0$ corresponds to isotropic expansion. The space approaches isotropy, in case of diagonal energy momentum tensor ( $T^{0 i}=0$, where $i=1,2,3$ ) if $\Delta \rightarrow 0, V \rightarrow \infty$ and $T^{00}>0 \quad(\rho>0)$ as $t \rightarrow \infty$ (Collins and Hawking, 1973).

Using equations (11) and (12,) equation (14) simplifies to

$$
\Delta=\frac{2}{9 H^{2}}\left(H_{x}-H_{y}\right)^{2},
$$

where $\left(H_{x}-H_{y}\right)$ is the difference between the expansion rates on $\mathrm{x}$ and $\mathrm{y}$ axes which can be obtained by using the field equations.

On subtracting (7) from equation (8), we obtain

$$
H_{x}-H_{y}=\frac{\dot{a}}{a}-\frac{\dot{b}}{b}=\frac{\lambda}{V}+\frac{1}{V} \int\left(\frac{b^{2}-a^{2}}{b^{4}}-\gamma \rho^{(d e)}\right) V d t,
$$

where $\lambda$ is a constant of integration and the term with $\gamma$ arises due to the possible intrinsic anisotropy of the fluid.

To obtain the anisotropy parameter of the expansion, using (16) in (15), we get

$$
\Delta=\frac{2}{9 H^{2}}\left(\lambda+\int\left(\frac{b^{2}-a^{2}}{b^{4}}-\gamma \rho^{(d e)}\right) V d t\right)^{2} V^{-2} .
$$

Choosing $\gamma=0$, the anisotropy parameter of the expansion for a Bianchi type-IX cosmological model in the presence of $\mathrm{PF}$ reduces to

$$
\Delta=\frac{2}{9 H^{2}}\left(\lambda+\int\left(\frac{b^{2}-a^{2}}{b^{4}}\right) V d t\right)^{2} V^{-2} .
$$

The integral term in (17) vanishes for

$$
\gamma=\frac{b^{2}-a^{2}}{\rho^{(d e)} b^{4}} \text {. }
$$

The energy momentum tensor (5) for anisotropic DE becomes

$$
T_{i}^{j}=\operatorname{diag}\left[-1, \omega^{(d e)}, \omega^{(d e)}+\frac{b^{2}-a^{2}}{\rho^{(d e)} b^{4}}, \omega^{(d e)}+\frac{b^{2}-a^{2}}{\rho^{(d e)} b^{4}}\right] \rho^{(d e)} .
$$

Using (19) in equation (17), the anisotropic parameter of the expansion reduces to

$$
\Delta=\frac{2}{9} \frac{\lambda^{2}}{H^{2}} V^{-2} \text {. }
$$

It is seen that the above anisotropic parameter of the expansion is exactly same for exponential expansion with anisotropic fluid $[16,33]$ and is equivalent to ones obtained for exponential expansion with isotropic fluid [39-40].

The vanishing of the integral term also reduces the difference between the expansion rates on $\mathrm{x}$ and $\mathrm{y}$ to the following form 


$$
H_{x}-H_{y}=\frac{\lambda}{V}=\frac{\lambda}{a b^{2}} \text {. }
$$
equations as

Using equations (19) and (20) in the Einstein's field equations (6-8), one can obtain the reduced field

$$
\begin{aligned}
& 2 \frac{\dot{a} \dot{b}}{a b}+\frac{\dot{b}^{2}}{b^{2}}+\frac{3 a^{2}}{4 b^{4}}=\rho^{(p f)}+(1-\gamma) \rho^{(d e)} \\
& 2 \frac{\ddot{b}}{b}+\frac{\dot{b}^{2}}{b^{2}}+\frac{1}{b^{2}}-\frac{3 a^{2}}{4 b^{4}}=-\omega^{(p f)} \rho^{(p f)}-\omega^{(d e)} \rho^{(d e)} \\
& \frac{\ddot{a}}{a}+\frac{\ddot{b}}{b}+\frac{\dot{a}}{a} \frac{\dot{b}}{b}+\frac{1}{b^{2}}-\frac{3 a^{2}}{4 b^{4}}=-\omega^{(p f)} \rho^{(p f)}-\omega^{(d e)} \rho^{(d e)} .
\end{aligned}
$$

\section{Solutions Of The Field Equations:}

Now we have, three linearly independent equations (23-25) and six unknown functions $a, b, \omega^{(p f)}$, $\omega^{(d e)}, \rho^{(p f)}, \rho^{(d e)}$. Hence, we need three extra equations to solve the system completely.

Firstly, we assume that the DE and PF is minimally interacting (Adhav [33]) i.e.

$$
{ }^{p f} T_{; j}^{i j}=0 \text { and }{ }^{d e} T_{; j}^{i j}=0
$$

Hence, the Bianchi identity (10) can be split into two separately additive conserved components. Therefore, the conservation equation of energy momentum tensor of DE and PF becomes

$$
\begin{aligned}
& { }^{d e} T_{; j}^{i j}=\dot{\rho}^{(d e)}+\left(1+\omega^{(d e)}\right) \rho^{(d e)}\left(\frac{\dot{a}}{a}+2 \frac{\dot{b}}{b}\right)+2 \gamma \rho^{(d e)} \frac{\dot{b}}{b}=0 . \\
& { }^{p f} T_{; j}^{i j}=\dot{\rho}^{(p f)}+\left(1+\omega^{(p f)}\right) \rho^{(p f)}\left(\frac{\dot{a}}{a}+2 \frac{\dot{b}}{b}\right)=0 .
\end{aligned}
$$

Secondly, we assume that the EoS parameter of the PF to be constant i.e.

$$
\omega^{(p f)}=\frac{p^{(p f)}}{\rho^{(p f)}}=\text { constant. }
$$

Lastly, we use respectively the exponential volumetric expansion law as

$$
V=C_{1} e^{3 k t}
$$

and the power law volumetric expansion as

$$
V=C_{1} t^{3 m}
$$

where $C_{1}, k$ and $m$ are positive constants.

For $m>1$, the model exhibits accelerating volumetric expansion, on the other hand for $m=1$, the model exhibits volumetric expansion with constant velocity whereas for $m<1$, the model exhibits decelerating volumetric expansion. The anisotropic fluid we take here can be considered in the context of DE in the model with exponential expansion and power law expansion for $m>1$.

\section{Model For Exponential Expansion $\mathrm{V}=\mathrm{C}_{1} \mathrm{e}^{3 \mathrm{kt}}$ :}

The scale factors $a$ and $b$ are obtained by solving the field equations (23-25) for the exponential volumetric expansion (29) by considering (22),

$$
\begin{aligned}
& a=\left(C_{1} C_{2}^{2}\right)^{1 / 3} \exp \left(k t-\frac{2}{9} \frac{\lambda}{k C_{1}} e^{-3 k t}\right), \\
& \text { (31) } b=\left(\frac{C_{1}}{C_{2}}\right)^{1 / 3} \exp \left(k t+\frac{1}{9} \frac{\lambda}{k C_{1}} e^{-3 k t}\right),
\end{aligned}
$$

where $C_{2}$ is a positive constant of integration.

The mean Hubble parameter is given by 


$$
H=k \text {. }
$$

The directional Hubble parameters on the $\mathrm{x}, \mathrm{y}$ and $\mathrm{z}$ axes are respectively are given by,

$$
H_{x}=k+\frac{2}{3} \frac{\lambda}{C_{1}} e^{-3 k t} \text { and } \quad H_{y}=H_{z}=k-\frac{1}{3} \frac{\lambda}{C_{1}} e^{-3 k t} .
$$

Using equations (33), (34) in equation (15), the anisotropic parameter of the expansion becomes,

$$
\Delta=\frac{2}{9} \frac{\lambda^{2}}{k^{2} C_{1}^{2}} e^{-6 k t} \text {. }
$$

It is observed that the above anisotropic parameter of the expansion is exactly same for exponential expansion with anisotropic fluid $[16,33]$ and is equivalent to ones obtained for exponential expansion with isotropic fluid [39-40].

Using equations (12) and (33) in equation (27), the energy density of the PF is found to be

$$
\rho^{(p f)}(t)=\rho_{0} e^{-3 k\left(1+\omega^{(p f)}\right) t} .
$$

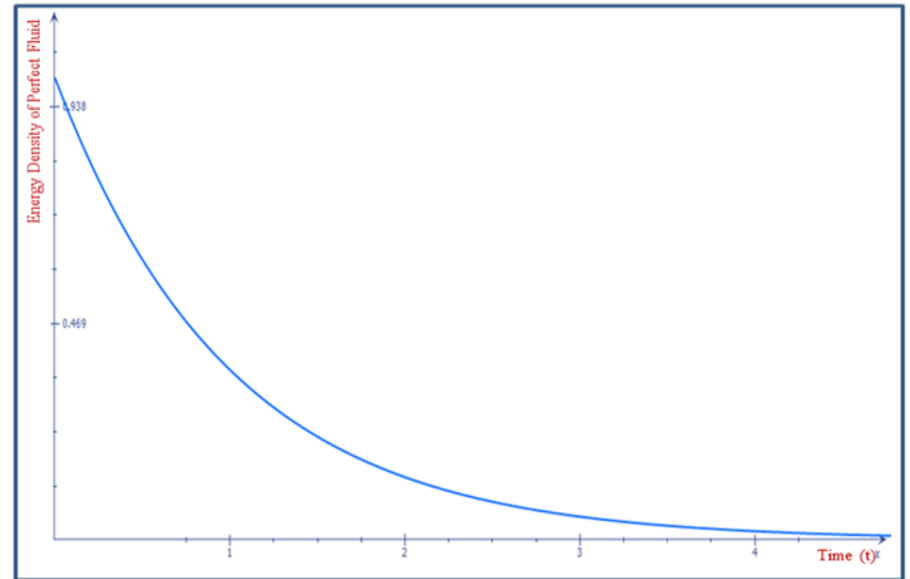

Figure 1. The Plot of Energy Density of Perfect Fluid $\left(\rho^{(\mathrm{PF})}\right)$ verses time $(t)$

From equation (36), it is observed that, the energy density of PF is constant at initial epoch and starts decreasing with increase in time and becomes zero as $t \rightarrow \infty$ as shown in figure 1 .

Using equations (31), (32) and (36) in equation (23), the energy density of the DE is found to be

$\rho^{(d e)}=3 k^{2}-\frac{\lambda^{2}}{3 C_{1}^{2}} e^{-6 k t}+\left(\frac{C_{2}}{C_{1}}\right)^{2 / 3} e^{-2 k t-\frac{2}{9} \frac{\lambda}{k C_{1}} e^{-3 k t}}-\frac{1}{4} C_{2}^{2}\left(\frac{C_{2}}{C_{1}}\right)^{2 / 3} e^{-2 k t-\frac{8}{9} \frac{\lambda}{k C_{1}} e^{-3 k t}}-\rho_{0} e^{-3 k\left(1+\omega^{(p f)}\right) t}$.

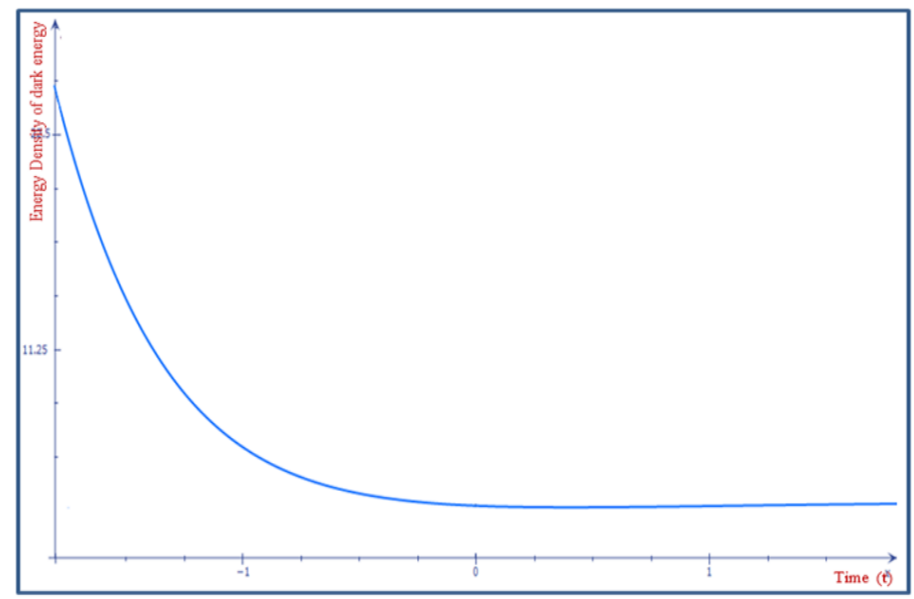

Figure 2. The Plot of Energy Density of Dark Energy $\left(\rho^{(\mathrm{de})}\right)$ verses time $(t)$

From equation (37), the energy density of the DE is constant at initial epoch and starts decreasing with increase in time. For large values of time $t$, the energy density of DE reduces to a fixed constant which matches with the data from recent cosmological observations that the universe is accelerating as shown in figure 2 . 
Using equations (31), (32) and (37) in equation (24), the deviation-free part of the anisotropic EoS parameter is obtained as

$$
\omega^{(d e)}=-\frac{3 k^{2}+\frac{\lambda^{2}}{3 C_{1}^{2}} e^{-6 k t}+\left(\frac{C_{2}}{C_{1}}\right)^{2 / 3} e^{-2 k t-\frac{2}{9} \frac{\lambda}{k C_{1}} e^{-3 k t}}-\frac{3}{4} C_{2}^{2}\left(\frac{C_{2}}{C_{1}}\right)^{2 / 3} e^{-2 k t-\frac{8}{9} \frac{\lambda}{k C_{1}} e^{-3 k t}}+\rho_{0} \omega^{(p f)} e^{-3 k\left(1+\omega^{(p f)}\right) t}}{3 k^{2}-\frac{\lambda^{2}}{3 C_{1}^{2}} e^{-6 k t}+\left(\frac{C_{2}}{C_{1}}\right)^{2 / 3} e^{-2 k t-\frac{2}{9} \frac{\lambda}{k C_{1}} e^{-3 k t}}-\frac{1}{4} C_{2}^{2}\left(\frac{C_{2}}{C_{1}}\right)^{2 / 3} e^{-2 k t-\frac{8}{9} \frac{\lambda}{k C_{1}} e^{-3 k t}}-\rho_{0} e^{-3 k\left(1+\omega^{(p f)}\right) t}}
$$

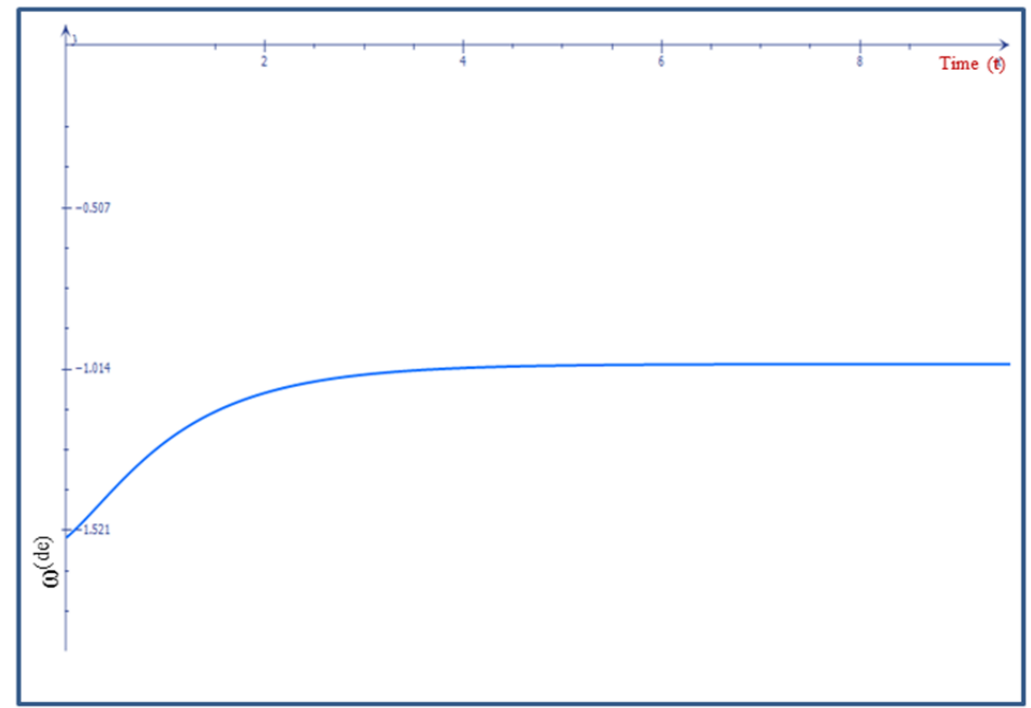

Figure 3. The Plot of $\omega^{(\mathrm{de})}$ verses time $(\mathrm{t})$

From equation (38), from initial epoch to some finite value of time t, the EoS parameter of the DE $\omega^{(d e)}$ is less than -1 i.e. in this region the model behaves like phantom dark energy universe. For increasing values of time, the dark energy behaves like a cosmological constant for which $\omega^{(d e)}=-1$ as shown in figure 3.

Also using equations (31), (32) and (37) in equation (19), the deviation skewness parameter $\gamma$ is obtained as

$$
\gamma=-\frac{C_{2}^{2}\left(\frac{C_{2}}{C_{1}}\right)^{2 / 3} e^{-2 k t-\frac{8}{9} \frac{\lambda}{k C_{1}} e^{-3 k t}}-\left(\frac{C_{2}}{C_{1}}\right)^{2 / 3} e^{-2 k t-\frac{2}{9} \frac{\lambda}{k C_{1}} e^{-3 k t}}}{3 k^{2}-\frac{\lambda^{2}}{3 C_{1}^{2}} e^{-6 k t}+\left(\frac{C_{2}}{C_{1}}\right)^{2 / 3} e^{-2 k t-\frac{2}{9} \frac{\lambda}{k C_{1}} e^{-3 k t}}-\frac{1}{4} C_{2}^{2}\left(\frac{C_{2}}{C_{1}}\right)^{2 / 3} e^{-2 k t-\frac{8}{9} \frac{\lambda}{k C_{1}} e^{-3 k t}}-\rho_{0} e^{-3 k\left(1+\omega^{(p f)}\right) t}}
$$

From equation (35), the anisotropy of the expansion $(\Delta)$ is not promoted by the anisotropy of the fluid and decreases to null exponentially as $\mathrm{t}$ increases. The space approaches to isotropy in this model as $\Delta \rightarrow 0$, $V \rightarrow \infty$ and $\rho=3 k^{2}>0$ as $t \rightarrow \infty$. The energy density $(\rho)$, the deviation free EoS parameter $(\omega)$ and the skewness parameter $(\gamma)$ are dynamical. Also there is no big-bang type of singularity for particular choice of parameters. As $t \rightarrow \infty$, the anisotropic fluid isotropizes and mimics the vacuum energy which is mathematically equivalent to the cosmological constant $(\Lambda)$ i.e. as $t \rightarrow \infty$ we get $\gamma \rightarrow 0, \omega \rightarrow-1$ and $\rho \rightarrow 3 k^{2}$ as in figure 1,2 and 3 respectively.

From equation (1), the statefinder parameters are found as

$$
r=1 \text { and } s=0
$$

which are similar to the $\Lambda \mathrm{CDM}$ cosmological model for which the statefinder parameters are $\{r, s\}=\{1,0\}$. 
According to the conditions given by Collins and Hawking [41], the model isotropizes for large values of $t$. Also, the anisotropy of the DE isotropizes for large values of $t$. This is consistent with the present day observations that the universe is isotropic.

\section{Model For Power Law Expansion $V=C_{1} t^{3 m}$ :}

The scale factors $a$ and $b$ are obtained by solving the field equations (23-25) for the Power Law volumetric expansion (30) by considering (22),

$$
\begin{aligned}
& a=\left(C_{1} C_{2}^{2}\right)^{1 / 3} t^{m} e^{\frac{2}{3} \frac{\lambda t_{1} \frac{1-3 m}{1-3 m}}{C_{2}}}, \\
& b=\left(\frac{C_{1}}{C_{2}}\right)^{1 / 3} t^{m} e^{-\frac{\lambda}{3 C_{1}} \frac{t^{1-3 m}}{1-3 m}},
\end{aligned}
$$

where $C_{1}, C_{2}$ are positive constants of integration.

The mean Hubble parameter is

$$
H=\frac{m}{t} \text {. }
$$

The directional Hubble parameters on the $\mathrm{x}, \mathrm{y}$ and $\mathrm{z}$ axes are respectively are given by

$$
H_{x}=\frac{m}{t}+\frac{2}{3} \frac{\lambda}{C_{1}} t^{-3 m} \quad \text { and } \quad H_{y}=H_{z}=\frac{m}{t}-\frac{1}{3} \frac{\lambda}{C_{1}} t^{-3 m} .
$$

Using equations (42), (43) in equation (15), the anisotropic parameter of the expansion becomes,

$$
\Delta=\frac{2}{9} \frac{\lambda^{2}}{C_{1}^{2}} \frac{t^{2-6 m}}{m^{2}} .
$$

Using equations (12) and (42) in equation (27), the energy density of the PF is found to be

$$
\rho^{(p f)}(t)=\rho_{0} t^{-3 m\left(1+\omega^{(p f)}\right)} \text {. }
$$

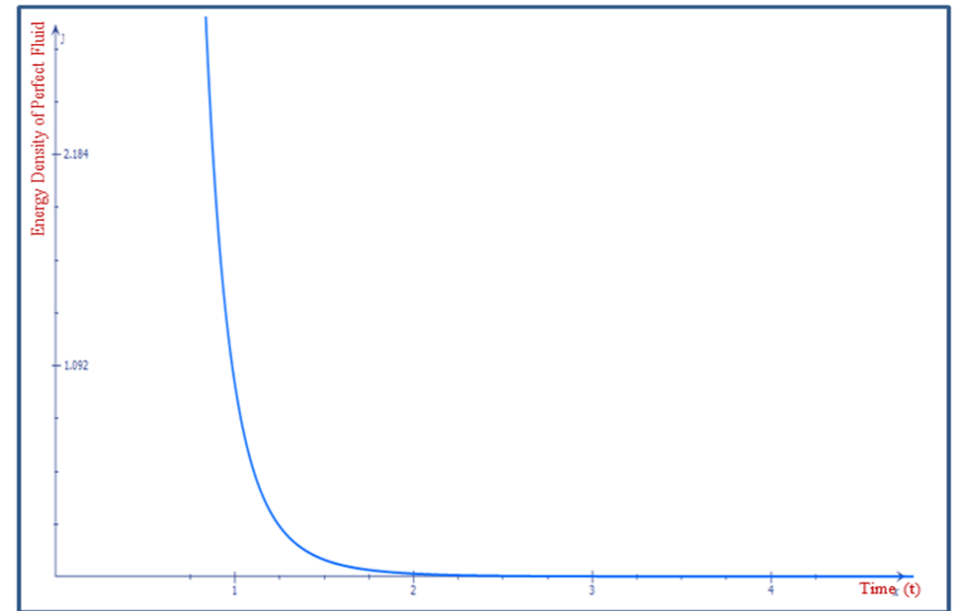

Figure 4. The Plot of Energy Density of Perfect Fluid $\left(\rho^{(\mathrm{PF})}\right)$ verses time $(t)$

From equation (45), it is observed that, the energy density of PF $\rho^{(p f)} \rightarrow \infty$ as $t \rightarrow 0$ i.e. there is a big-bang singularity and starts decreasing with increase in time and becomes zero as $t \rightarrow \infty$ as shown in fig. 4 . Using equations (40), (41) and (45) in equation (23), the energy density of the DE is found to be

$$
\rho=3 m^{2} t^{-2}-\frac{\lambda^{2}}{3 C_{1}^{2}} t^{-6 m}+\left(\frac{C_{2}}{C_{1}}\right)^{2 / 3} t^{-2 m} e^{-\frac{2}{3} \frac{\lambda}{C_{1}} \frac{t^{1-3 m}}{1-3 m}}-\frac{1}{4} C_{2}^{2}\left(\frac{C_{2}}{C_{1}}\right)^{2 / 3} t^{-2 m} e^{-\frac{8}{3} \frac{\lambda}{C_{1}} \frac{t^{1-3 m}}{1-3 m}}-\rho_{0} t^{-3 m\left(1+\omega^{(p f)}\right)}
$$




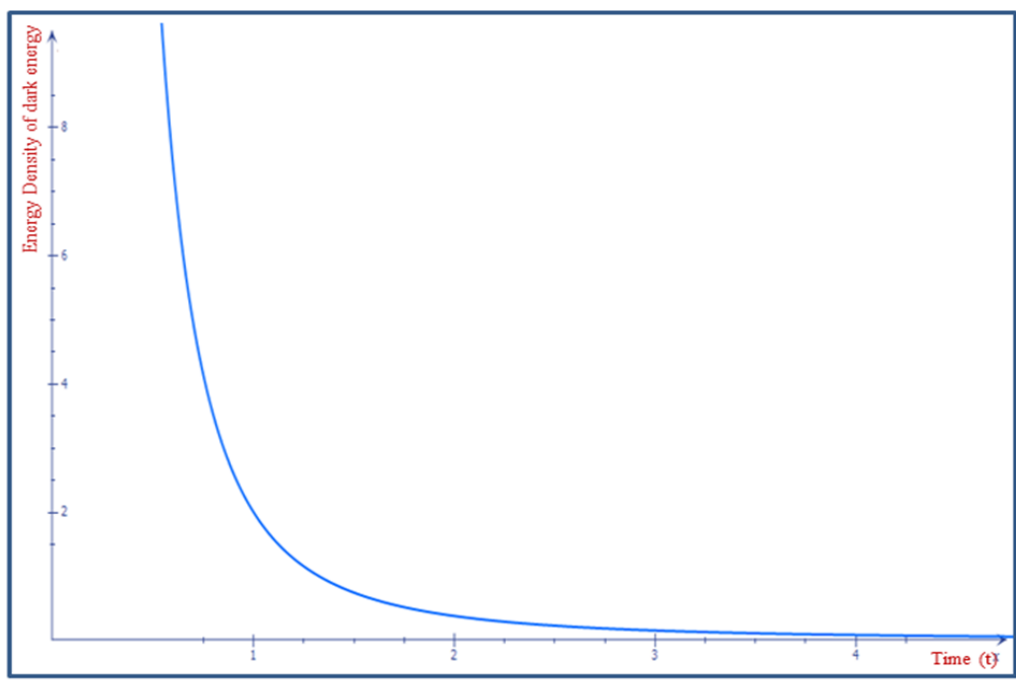

Figure 5. The Plot of Energy Density of Dark Energy $\left(\rho^{(\mathrm{de})}\right)$ verses time $(t)$

From equation (46), the energy density of the DE $\rho^{(d e)} \rightarrow \infty$ as $t \rightarrow 0$ and starts decreasing with increase in time. For large values of time $t$, the energy density of DE reduces and becomes zero as $t \rightarrow \infty$ as shown in fig. 5.

Using equations (40), (41) and (42) in equation (24), the deviation free part of the anisotropic EoS parameter is obtained as

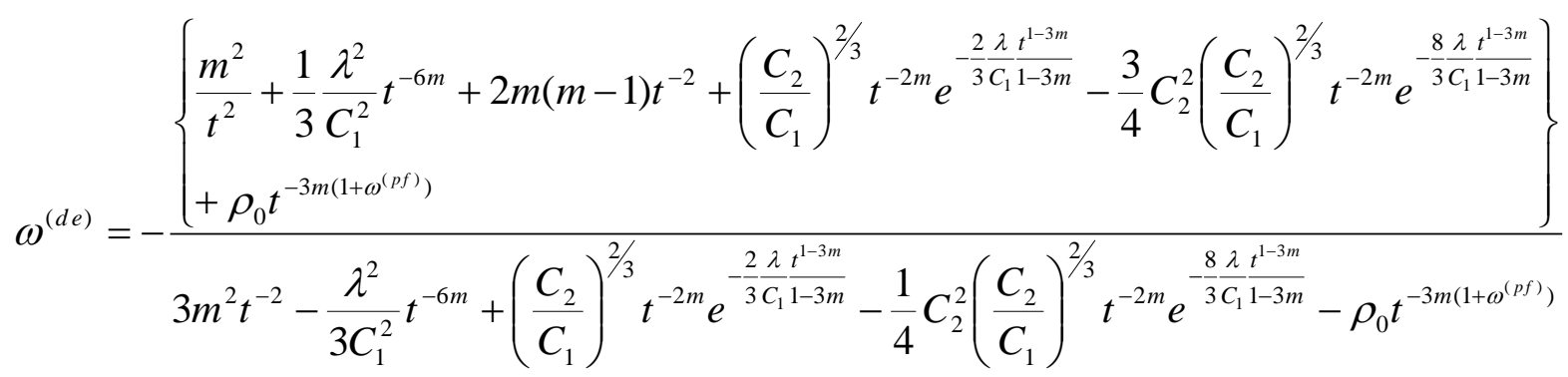

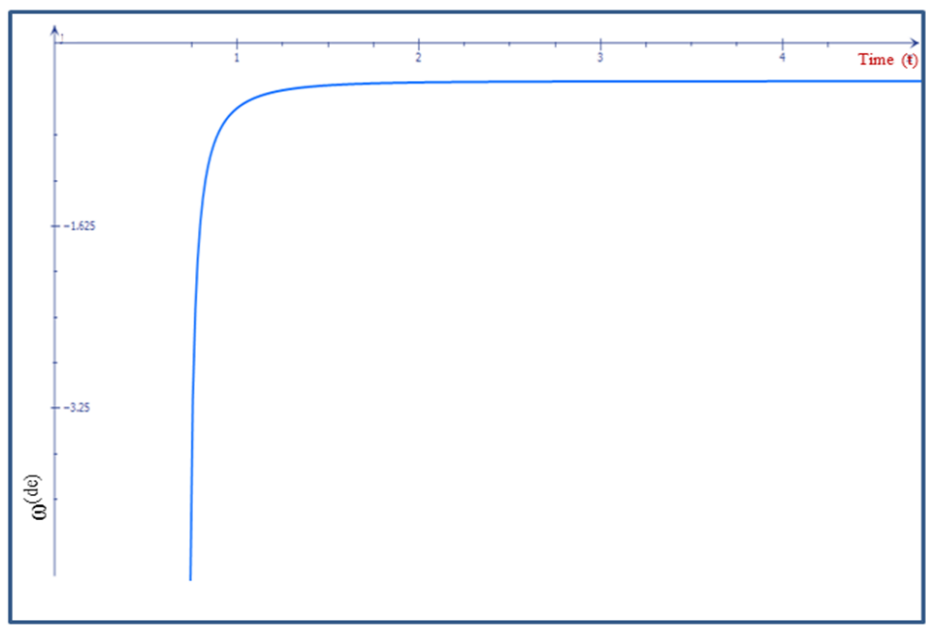

Figure 6. The Plot of $\omega^{(\mathrm{de})}$ verses time (t)

From equation (47), at initial epoch to some finite value of time $t$, the EoS parameter of the DE is less than $-1\left(\omega^{(d e)}<-1\right)$ i.e. in this region the model behaves like phantom dark energy universe. For increasing values of time, the dark energy behaves like a cosmological constant for which $\omega^{(d e)}=-1$ as shown in fig. 6 . 
Also using equations (40), (41) and (42) in equation (19), the deviation skewness parameter $\gamma$ on $\mathrm{z}$ axis is obtained as

$$
\gamma=-\frac{C_{2}^{2}\left(\frac{C_{2}}{C_{1}}\right)^{2 / 3} t^{-2 m} e^{-\frac{8}{3} \frac{\lambda}{C_{1}} \frac{t^{1-3 m}}{1-3 m}}-\left(\frac{C_{2}}{C_{1}}\right)^{2 / 3} t^{-2 m} e^{-\frac{2}{3} \frac{\lambda}{C_{1}} \frac{t^{1-3 m}}{1-3 m}}}{3 m^{2} t^{-2}-\frac{\lambda^{2}}{3 C_{1}^{2}} t^{-6 m}+\left(\frac{C_{2}}{C_{1}}\right)^{2 / 3} t^{-2 m} e^{-\frac{2}{3} \frac{\lambda}{C_{1} \frac{t^{1-3 m}}{1-3 m}}}-\frac{1}{4} C_{2}^{2}\left(\frac{C_{2}}{C_{1}}\right)^{2 / 3} t^{-2 m} e^{-\frac{8}{3} \frac{\lambda t_{1} \frac{t^{1-3 m}}{1-3 m}}{C_{0}}}-\rho_{0} t^{-3 m\left(1+\omega^{(p f)}\right)}}
$$

From equation (42), it is observed that, the mean Hubble parameter $H$ is infinitely large at $t=0$ and reduces for increasing values of $\mathrm{t}$ and becomes zero as $t \rightarrow \infty$. The anisotropy of the expansion $(\Delta)$ behaves monotonically, decays to zero for $m>1 / 3$ and diverges for $m<1 / 3$ and is constant for $m=1 / 3$.

\section{Statefinder Diagnostic}

In the last few years number of cosmologists have developed more number of DE models. To explain the current cosmic acceleration the oldest and most familiar method is by observing the Hubble constant $H_{0}$ and the current value of the deceleration parameter $q_{0}$. Sahni et al. [ ] and Alam et al. [ ] have proposed a new method to explain the current cosmic acceleration by introducing a cosmological diagnostic pair $\{r, s\}$ which they termed as statefinder. The two parameters are dimensionless and are geometrical since they are derived from cosmic scale factors alone, though one can rewrite them in terms of the parameters of DE and DM. This pair gives information about DE in a model-independent way i.e. it categorizes DE in the context of background geometry only which is not dependent on theory of gravity. The statefinder parameters are depends upon the expansion factor and hence upon the metric describing space and time. They can effectively differentiate different forms of DE and provide simple diagnosis regarding whether a particular model fits into the basic observational data. According to Sahni, the statefinder parameters $\{r, s\}$ are defined as

$$
r(t)=\frac{\dddot{R}}{R H^{3}} \quad \text { and } \quad s(t)=\frac{r-1}{3\left(q-\frac{1}{2}\right)}
$$

where $H$ is the Hubble parameter, $R$ is an average scale factor and $q$ is the deceleration parameter.

In this letter, models for exponential expansion and power law expansion are discussed. In the former model, we observed that the statefinder parameters are found as $r=1$ and $s=0$ which are similar to the $\Lambda \mathrm{CDM}$ cosmological model for which the statefinder parameters $\{r, s\}=\{1,0\}$ indicating that the model isotropizes for large values of $\mathrm{t}$. Also the anisotropy of DE isotropizes for large value of $\mathrm{t}$ which are consistent with the present day observations that the universe is isotropic. In the latter model the statefinder parameters are found to be

$$
r(t)=\frac{\dddot{R}}{R H^{3}}=\frac{(m-1)(m-2)}{m^{2}} \quad \text { and } \quad s(t)=\frac{r-1}{3\left(q-\frac{1}{2}\right)}=\frac{2 m(r-1)}{3(m-2)} .
$$

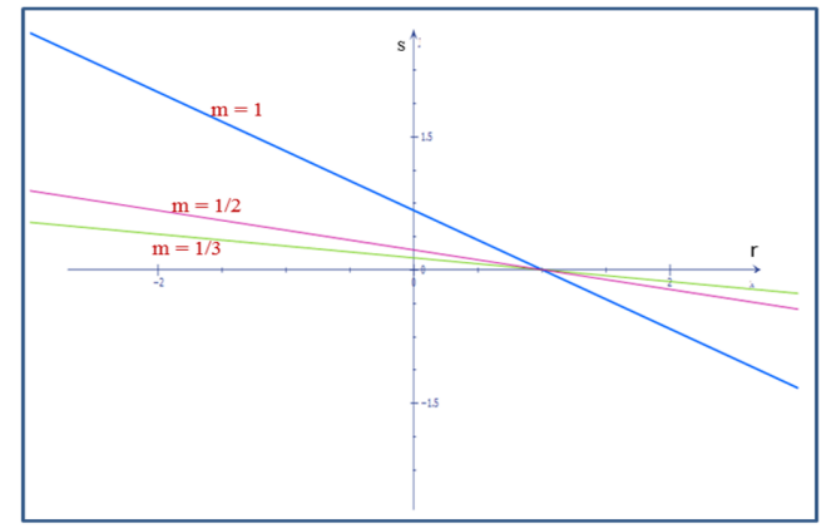

Figure 7. The Plot of variation $\mathrm{r}$ against $\mathrm{s}$ for different values of $(\mathrm{m}=1,1 / 2,1 / 3)$ 
Fig. 7 shows the variation of $r$ against $s$ for the values of $m=\frac{1}{3}, \frac{1}{2}, 1$. We observe that the model starts from an Einstein Static Era $(r \rightarrow \infty, s \rightarrow-\infty)$ and goes to the $\Lambda$ CDM model $(r=1, s=0)$.

\section{Conclusion}

The new Bianchi type-IX cosmological models is obtained with PF and anisotropic DE on the basis of exponential expansion and power law expansion. In both the models the anisotropic DE isotropizes for large values of $t$. The solution of the field equations are obtained by using special law for the mean Hubble parameter (Berman, 1983) which yields a constant value of the deceleration parameter. The anisotropy of the space isotropizes for exponential expansion. Also the anisotropy of the space isotropizes for power law expansion model provided $m>1 / 3$. The statefinder diagnostic pair i.e. $\{r, s\}$ parameter is adopted to characterize different phases of the universe. It is observed that the exponential expansion model is similar to $\Lambda \mathrm{CDM}$ cosmological model for which the statefinder parameters are $\{r, s\}=\{1,0\}$. In case of power law expansion model, the evolution trajectories in statefinder $r-s$ plane are plotted in figure 7. The power law expansion model has the counters in the $r-s$ plane which shows the different phases of the universe. It is interesting to note that our observations are analogous to the investigations of Akarsu and Kilinc [42], Adhav [34] and Ghate and Sontakke [16].

\section{References}

[1] S. Perlmutter et al., "Discovery of Supernovae explosion at half the age of the Universe", Nature, Vol. 391, No. 2, pp. 51-54, 1998. doi: $10.1038 / 34124$

[2] A. G. Reiss, et al., "Observational Evidence from super-novae for an Accelerating Universe and a Cosmological Constant," The Astrophysical Journal, Vol. 116, No. 3, 1998, pp. 1009-1038. doi:10.1086/300499.

[3] C. L. Benett et al., "First-Year Wilkinson Microwave Anisotropy Probe (WMAP) Observations: Preliminary Maps and Basic Results", Astrophys. J. Suppl. Ser., Vol. 148, No. 1, pp. 1, 2003. doi:10.1086/377253

[4] Hawkins S. et al., "The 2dF galaxy redshift survey: correlation functions, peculiar velocities and the matter density of the universe", Monthly Notices of the Royal Astronomical Society, Vol. 346 No. 1, pp. 78-96, 2003. doi.10.1046/j.1365-2966.2003.07063.x

[5] R. R. CALDWEll, M. DORAN, "COSMIC MICROWAVE BACKGROUND AND SUPERNOVA CONSTRAINTS ON QUINTESSENCE: CONCORDANCE REgIONS AND TARget MODEls”, Physics REVIEW D, VOL. 69, No. 10, $103517,2004 . \quad \underline{\text { DOI. }}$ 10.1103/PHYSREVD.69.103517.

[6] Z. Y. HUANG, B. WANG, E. ABDALLA, "HOLOGRAPHIC EXPLANATION OF WIDE-ANGLE POWER CORRELATION SUPPRESSION IN THE COSMIC MICROWAVE BACKGROUND RADIATION”, JOURNAL OF COSMOLOGY AND ASTROPARTICLE PHYSICS, VOL. 2006, NO. 05, DOI: $10.1088 / 1475-7516 / 2006 / 05 / 013$

[7] S. D. TADE, M. M. SAMBHE, "BIANCHI TYPE-I COSMOLOGICAL MODELS FOR BINARY MIXTURE OF PERFECT FLUID AND DARK ENERGY”, ASTROPHYSICS AND SPACE SCIENCE, VOL. 338, PP. 179-185, 2012. DOI: 10.1007/S10509-011-0910-8

[8] S. KUMAR, O. AKARSU, "BIANCHI TYPE-II MODELS IN THE PRESENCE OF PERFECT FLUID AND DARK ENERGY", THE EUROPEAN PHYSICAL JOURNAL PLUS, VOL. 127, PP. 64, 2012. DOI: 10.1110/EPJP/I2012-12064-4

[9] S. D. KATORE ET AL., "BIANCHI TYPE-III COSMOLOGICAL MODEL WITH BINARY MIXTURE OF PERFECT FLUID AND DARK ENERGY", BULGARIAN JOURNAL OF PHYSICS, VOL. 38, PP. 390-399, 2011.

[10] T. SiNGH, R. CHAUBEY, "BIANCHI TYPE-V COSMOLOGICAL MODELS WITH PERFECT FLUID AND DARK ENERGY", ASTROPHYSICS AND SPACE SCIENCE, VOL. 319, PP. 149-154, 2009. DOI: 10.1007/S10509-008-9959-4

[11] KATORE ET AL., "BIANCHI TYPE-VI ${ }_{0}$ COSMOLOGICAL MODELS WITH PERFECT FLUID AND DARK ENERGY", INTERNATIONAL JOURNAL OF THEORETICAL PHYSICS, VOL. 50, PP. 3299-3312, 2011B. DOI: 10.1007/s10773-011-0832-9

[12] S. D. Katore, K. S. Adhav, A. Y. ShaikH, M. M. SANChETI, "Plane SYMMETRIC COSMOLOGICAL MODELS With PERFECT Fluid AND DARK ENERGY”, ASTROPHYSICS AND SPACE SCIENCE, VOL. 333, PP. 333-341, 2011C. DOI: 10.1007/S10509-011-0622-0

[13] S. D. KATORE ET AL., "KALUZA-KLEIN COSMOLOGICAL MODEL FOR PERFECT FLUID AND DARK ENERGY", BULGARIAN JOURNAL OF PHYSICS, VOL. 40, PP. 17-32, 2013.

[14] K. S. Adhav et al., "Higher dimensional Bianchi type-I universe with perfect fluid and dark energy", Bulgarian Journal of Physics, Vol. 37, pp. 255-265, 2010.

[15] Adhav et al., "Higher dimensional Bianchi type-V cosmological models with perfect fluid and dark energy", International Journal of Theoretical Physics, Vol. 50, pp. 2573-2581, 2011. doi: 10.1007/s10773-011-0751-9

[16] H. R. Ghate, A. S. Sontakke, "Bianchi type-IX cosmological models with a anisotropic dark energy", International Journal of Scientific and Engineering Research, Vol. 4, No. 6, pp. 769-774, 2013.

[17] S. Chakraborty, "A study on Bianchi-IX cosmological model,” Astrophysics and Space Science, Vol. 180, No. 2, pp. 293-303, 1991. doi: 10.1007/BF00648184

[18] Shri Ram, Prem Singh, "Bianchi type-II, VIII and IX cosmological models with matter and electromagnetic fields", Astrophysics and Space Science, Vol. 201, No. 1, pp. 29-33, 1993. doi:10.1007/BF00626970

[19] K. S. Adhav et al., "Axially Symmetric Bianchi type-IX Inflationary Universe in General Relativity", International Journal of Theoretical Physics, Vol. 49, pp. 1753-1758, 2010. doi:10.1007/s10773-010-0355-9

[20] S.M.Waller, "Bianchi type IX electromagnetic universes", Physical Review D., Vol. 29, No. 2, pp.176-185, 1984.

[21] R. Bali and S. Dave, "Bianchi Type-IX string cosmological model in general relativity," Pramana, vol. 56, no. 4, pp. 513-518, 2001.

[22] R. Bali and M. K. Yadav, "Bianchi Type-IX viscous fluid cosmological model in general relativity,” Pramana Journal of Physics, Vol. 64, No. 2, pp. 187-196, 2005. doi: 10.1007/BF02704873

[23] R. Bali, P. Kumawat, "Bianchi type IX stiff fluid tilted cosmological model with bulk viscosity", European Journal of Theoretical Physics, Vol. 7, No 24, pp. 383-394, 2011. 
[24] A. Pradhan, S. K. Srivastav, and M. K. Yadav, "Some homogeneous Bianchi type IX viscous fluid cosmological models with a varying $\Lambda$," Astrophysics and Space Science, vol. 298, pp. 419-432, 2005.

[25] A. Tyagi, D. Chhajed, "Homogeneous anisotropic Bianchi type-IX cosmological model for perfect fluid distribution with electromagnetic field," American Journal of Mathematics and Statistics, vol. 2, No. 3, pp. 19-21, 2012. doi: 10.5923/j.ajms.20120203.01

[26] H. R. Ghate, A. S. Sontakke, "Bianchi Type-IX Dark Energy Model in Brans-Dicke Theory of Gravitation," Prespacetime Journal, vol. 4, no. 4, pp. 366-376, 2013.

[27] V. Sahni et al., "Statefinder-A new geometrical diagnostic of dark energy", JETP Letters, Vol. 77, No. 5, pp. 201-206, 2003.

[28] U. Alam et al., "Exploring the expanding Universe and dark energy using the statefinder diagnostic", Mon. Not. Roy. Astron. Soc., Vol. 344, No. 4, pp. 1057-1074, 2003.

[29] E. O. Ishida, "Statefinder Revisited", Brazilian Journal of Physics, Vol. 35, No. 4B, pp. 1172-1178, 2005.

[30] Y. Shao, "Statefinder parameters for Tachyon Dark Energy Model", Modern Physics Letters A, Vol. 23, No. 1, pp. 65, 2008. doi:10.1142/S0217732308023499

[31] M. Jamil, "Variable G correction to Statefinder Parameters of dark energy", International Journal of Theoretical Physics, Vol. 49, No. 11, pp. 2829-2840, 2010.

[32] M. R. Setare, M. Jamil, "Statefinder diagnostic and stability of modified gravity consistent with holographic and agegraphic dark energy”, General Relativity and Gravitation, Vol. 43, No. 1, pp. 293-303, 2011.

[33] K. S. Adhav, "Statefinder diagnostic for binary mixture of Anisotropic dark energy and Perfect fluid in Bianchi type-III Universe", Prespacetime Journal, Vol. 2, No. 9, pp. 1353-1368, 2011

[34] K. S. Adhav, "Statefinder diagnostic for Variable Modified Chaplygin Gas in LRS Bianchi type I Universe", Advances in Mathematical Physics, Vol. 2012, pp. 714350-714361, 2012. doi:10.1155/2012/714350

[35] S. D. Katore, A. Y. Shaikh, "Statefinder diagnosis for Modified Chaplygin Gas in Plane Symmetric Universe", The African Review of Physics, Vol. 7, pp. 0004, 2012.

[36] S. Chakraborty et al., "Statefinder parameters for different models with variable G correction in Kaluza-Klein cosmology", International Journal of Theoretical Physics, Vol. 51, No. 7, pp. 2246-2255, 2012. doi:10.1007/s10773-012-1194-Z

[37] M. Malekjani, A. Khodam-Mohammadi, "Statefinder diagnosis and the interacting ghost model of dark energy", Astrophysics and Space Science, Vol. 343, No. 1, pp. 451-461, 2013.

[38] Y. Fei, Z. Jing-Fei, "Statefinder diagnosis for the Extended Holographic Ricci Dark Energy Model without and with interaction.", Communications in Theoretical Physics, Vol. 59, No. 2, pp. 243, 2013. doi:10.1088/0253-6102-/59/2/17

[39] S. Kumar, C. P. Singh, "Anisotropic Bianchi type-I models with constant deceleration parameter in general relativity", Astrophysics and Space Science, Vol. 312, No. 1, pp. 57-62, 2007. doi:10.1007/s10509-007-9623-4

[40] C. P. Singh, Shri Ram, M. Zeyauddin, "Bianchi type-V perfect fluid space-time in general relativity", Astrophysics and Space Science, Vol. 315, No. 1, pp. 181-189, 2008. doi:10.1007/s10509-008-9811-X

[41] C. B. Collins, S. W. Hawking, "Why is the Universe Isotropic", The Astrophysical Journal, Vol. 180, pp. 317-334, 1973. doi: $10.1086 / 151965$

[42] O. Akarsu, C. B. Kilinc, "LRS Bianchi type I models with Anisotropic Dark Energy and Constant Deceleration Parameter", General Relativity and Gravitation, Vol. 42, No. 1, pp. 119-140, 2010. doi:10.1007/s10714-009-0821-y 